Western University

Scholarship@Western

Brain and Mind Institute Researchers'

Publications

Brain and Mind Institute

$10-1-2013$

\title{
The touchscreen operant platform for testing working memory and pattern separation in rats and mice.
}

Charlotte A Oomen

Department of Psychology, University of Cambridge, Cambridge, UK \& Behavioural and Clinical Neuroscience Institute, University of Cambridge, Cambridge, UK

Martha Hvoslef-Eide

Department of Psychology, University of Cambridge, Cambridge, UK \& Behavioural and Clinical Neuroscience Institute, University of Cambridge, Cambridge, UK

Christopher J Heath

Department of Psychology, University of Cambridge, Cambridge, UK \& Behavioural and Clinical Neuroscience Institute, University of Cambridge, Cambridge, UK

\section{Adam C Mar}

Department of Psychology, University of Cambridge, Cambridge, UK \& Behavioural and Clinical Neuroscience Institute, University of Cambridge, Cambridge, UK

Alexa E Horner

Department of Psychology, University of Cambridge, Cambridge, UK \& Behavioural and Clinical Neuroscience Institute, University of Cambridge, Cambridge, UK \& Synome Ltd., Moneta Building, Babraham Research Campus, Cambridge, UK

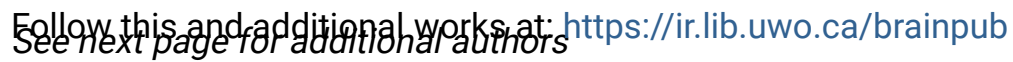

Part of the Neurosciences Commons, and the Psychology Commons

\section{Citation of this paper:}

Oomen, Charlotte A; Hvoslef-Eide, Martha; Heath, Christopher J; Mar, Adam C; Horner, Alexa E; Bussey, Timothy J; and Saksida, Lisa M, "The touchscreen operant platform for testing working memory and pattern separation in rats and mice." (2013). Brain and Mind Institute Researchers' Publications. 100. https://ir.lib.uwo.ca/brainpub/100 


\section{Authors}

Charlotte A Oomen, Martha Hvoslef-Eide, Christopher J Heath, Adam C Mar, Alexa E Horner, Timothy J Bussey, and Lisa M Saksida 


\title{
The touchscreen operant platform for testing working memory and pattern separation in rats and mice
}

\author{
Charlotte A Oomen 1,2 , Martha Hvoslef-Eide ${ }^{1,2}$, Christopher J Heath ${ }^{1,2}$, Adam C Marr,2, Alexa E Horner ${ }^{1-3}$, \\ Timothy J Bussey ${ }^{1,2} \&$ Lisa M Saksida ${ }^{1,2}$
}

\begin{abstract}
${ }^{1}$ Department of Psychology, University of Cambridge, Cambridge, UK. ${ }^{2}$ Behavioural and Clinical Neuroscience Institute, University of Cambridge, Cambridge, UK. ${ }^{3}$ Synome Ltd., Babraham Research Campus, Cambridge, UK. Correspondence should be addressed to C.A.O. (charlotte.oomen@gmail.com).
\end{abstract}

Published online 19 September 2013; doi:10.1038/nprot.2013.124

The automated touchscreen operant chamber for rats and mice allows for the assessment of multiple cognitive domains within the same testing environment. This protocol presents the location discrimination (LD) task and the trial-unique delayed nonmatchingto-location (TUNL) task, which both assess memory for location. During these tasks, animals are trained to a predefined criterion during $~ 20-40$ daily sessions. In LD sessions, touching the same location on the screen is rewarded on consecutive trials, followed by a reversal of location-reward contingencies. TUNL, a working memory task, requires animals to 'nonmatch' to a sample location after a delay. In both the LD and TUNL tasks, spatial similarity can be varied, allowing assessment of pattern separation ability, a function that is thought to be performed by the dentate gyrus (DG). These tasks are therefore particularly useful in animal models of hippocampal, and specifically DG, function, but they additionally permit discernment of changes in pattern separation from those in working memory.

\section{INTRODUCTION}

The touchscreen operant chamber platform for rats and mice enables presentation of a variety of cognitive tests, which in many cases are highly similar to touchscreen tasks used in human cognitive testing. Within this platform, several tasks have been developed that involve learning and memory of locations on the screen ${ }^{1-4}$. Of these, the TUNL task ${ }^{3}$ and the LD task ${ }^{1}$ are described here.

\section{Assessing spatial learning and memory}

The acquisition and demonstration of memory for locations has been extensively modeled in rodents. The focus has been on hippocampal function and pathology, which is often assessed using mazes such as the Morris water maze ${ }^{5}$, Barnes maze ${ }^{6}$, $\mathrm{T}$-maze and radial arm maze ${ }^{7,8}$. In these tasks, animals are trained to navigate, using distal cues, toward a place that is associated with a reinforcer (i.e., means of escape or food reward). Alternatively, automated tests in operant chambers have addressed memory for location using paradigms such as delayed matching or nonmatching-to-position (DNMTP) ${ }^{9,10}$. In these tests, animals are trained to sample one of two fixed locations by pressing a lever. After the so-called sample phase, a delay is imposed, during which the sample lever is retracted. At the end of the delay, the two levers are presented during the choice phase, and to obtain a food reward a matching or nonmatching rule needs to be applied. The touchscreen tasks described here are based on the same principle of using automated operant chambers to assess spatial learning and memory. It should be noted that operant chamber tests may not rely on spatial navigation per se, at least not in the same manner as maze tasks. However, operant chamber tests can be a valuable tool for detecting changes in the neural substrates underlying memory for location, such as the hippocampus ${ }^{11-13}$.

Advantages and limitations of the touchscreen platform Advantages of the use of the touchscreen platform have been discussed in detail elsewhere ${ }^{14-16}$, but a particular benefit of this method is that performance can be compared with other tasks carried out in the same testing environment, which facilitates comparison between tasks ${ }^{14,16-18}$. In addition, automated tests offer advantages such as standardized test procedures, minimized experimenter involvement and stress, lowered motor/mobility demands, simultaneous assessment of animals and improved sensitivity of measures such as response latencies.

With respect to potential limitations, touchscreen operant chamber tasks, as presented here and elsewhere ${ }^{16,18}$, make use of visual stimuli and are reward based, which may confound testing in certain animal models. More specifically, the use of visual stimuli precludes the use of certain subjects, such as mice with genetic alterations that cause rapid retinal degeneration. In addition, common albino rat or mouse strains differ in visual acuity when compared with pigmented strains ${ }^{19,20}$, although albino rodents appear to have sufficient vision to perform as well in the touchscreen as pigmented animals ${ }^{15}$. Finally, as with most appetitive operant paradigms, the use of food reward may introduce possible problems; for example, an experimental treatment may affect appetite or interact with the physiological effects of food restriction.

A particular advantage of the touchscreen, which is used in the TUNL and LD tasks, is the flexible nature of the screen, which allows the experimenter to manipulate the distance between, and therefore the similarity of, response locations. This provides a useful tool with respect to the functional contribution of the hippocampal DG, as it has been suggested that this region is a pattern separator of spatial information ${ }^{21-23}$. Pattern separation refers to the ability of neural circuits to orthogonalize or decorrelate similar input patterns into distinct representations ${ }^{24,25}$ to avoid memory interference. Behavioral evidence for the role of the DG as a pattern separator in spatial memory has been found in rats and mice ${ }^{23,26-28}$, and was initially obtained using a delayed matching-to-sample paradigm in a holeboard maze, in which the distance between choice locations was varied and DG lesions (but not lesions of the CA1, a different hippocampal subregion) 
resulted in impairments in recognizing similar, but not dissimilar, locations ${ }^{24}$. In the TUNL and LD tasks, a comparable approach is used with respect to measuring pattern separation. Specifically, both tests compare performance on a dissimilar condition (large separation) with that on a similar condition (small separation), aiming to place a variable demand on pattern separation capacity and designed to provide a behavioral readout of this computation. The dissimilar condition in particular allows for the dissociation of changes in pattern separation from general changes in location-memory and rule learning. These tasks may therefore be particularly useful in (disease) models that show changes in the DG, such as altered levels of adult hippocampal neurogenesis. Indeed, aberrant adult neurogenesis has been implicated in psychopathological and neurodegenerative diseases 29,30 such as depression ${ }^{31,32}$, schizophrenia ${ }^{33,34}$ and Alzheimer's disease ${ }^{35-38}$. It is changed by acute, chronic and developmental stress ${ }^{39-43}$, aging ${ }^{44,45}$, exercise ${ }^{46}$ and hormone levels ${ }^{47,48}$, among other factors, and it has been suggested to have a role in cognitive functions such as hippocampus-dependent learning and memory ${ }^{49-52}$.

\section{TUNL}

The TUNL task, developed by Talpos et al. ${ }^{3}$, is a working memory task based on the operant DNMTP paradigm with an important alteration: the predefined sample and novel choice location can be randomly selected from multiple response locations and vary between trials within a session, rendering this task more 'trial-unique.' Although the DNMTP task in a two-lever operant chamber has been found to be sensitive to both prefrontal and hippocampal lesions ${ }^{11,53,54}$ (compare to ref. 55), it has been criticized for allowing mediating strategies. Specifically, the requirement to retain spatial information across the delay can be reduced by taking advantage of the predictability of the tobe-correct location ${ }^{56-59}$. The TUNL task circumvents this issue by using an array of spatial locations, thereby making the to-becorrect choice location less predictable, and extensive analysis of putative mediating behaviors has shown that animals are unlikely to make use of such strategies ${ }^{3}$. An advantage of using multiple spatial locations is the ability to manipulate the distance between the sample and the to-be-correct location, so that in addition to the assessment of working memory, effects on pattern separation can be measured. Drawbacks of the TUNL task include a high task difficulty level, resulting in a slow acquisition rate. Furthermore, the task has so far been developed only for rats. Mice, in our experience, perform poorly on the TUNL task in its current form. However, we are addressing task difficulty through the development of a continuous version of the TUNL task that is acquired more rapidly (C.A.O., unpublished data), and we are currently developing a version for mice. The TUNL task as presented here has been validated as sensitive to hippocampal dysfunction, as lesioning this structure impairs performance in both a delay- and a separation-dependent manner ${ }^{3}$. In contrast, lesions to the prefrontal cortex impair performance when the delay, but not the separation, is manipulated, resulting in impairments under longdelay, but not short-delay conditions ${ }^{60}$. In addition to variations in delay, working memory capacity in TUNL may also be assessed by manipulating the degree of interference. In the current task setup, memory of earlier events may interfere with the memory of more recent events, causing proactive interference ${ }^{61}$. Inter-trial interference may be increased by shortening the inter-trial interval
(ITI ${ }^{62-64}$, and performance on increased interference probe sessions in the TUNL task was recently shown to be dependent on an intact prefrontal cortex ${ }^{60}$. To conclude, the TUNL task offers a paradigm in which both working memory and pattern separation requirements can be assayed with sufficient sensitivity to detect deficits in isolated processes. In light of this, the TUNL task may be particularly interesting for use in rodent models of disorders in which working memory deficits are a core feature of the phenotype, such as animal models of schizophrenia ${ }^{65,66}$. Furthermore, structural and functional changes in-and connectivity between-the hippocampus and prefrontal cortex have an important part in the pathophysiology of schizophrenia ${ }^{67-70}$.

\section{Location discrimination}

The LD task, developed by McTighe et al. ${ }^{1}$, was originally devised as a simplified procedure for studying pattern separation ability in rats and mice, and it was shown to be sensitive to hippocampal lesions. The protocol has been successfully implemented in both rats ${ }^{1}$ and (male, female and aged) mice $28,71,72$. In the LD task, two locations are presented on each trial, and the subject learns across trials to respond to the correct location. By using the LD task, pattern separation was shown to be dependent on adult neurogenesis in the $\mathrm{DG}^{28}$, and further studies have shown that the ability to pattern-separate in this task correlates with voluntary exercise levels and neurogenesis ${ }^{71}$, and that it is associated with glutamate receptor regulation and signaling 72 . Moreover, the current protocol includes a spatial reversal component, which could permit the discernment of changes in executive function from pattern separation per se. Potential issues with this task include the difficulty some subjects (depending on species and strain) may have in completing acquisition and reversal within a single session. However, the basic LD task protocol is easily amenable to modifications in experimental design to flexibly address the limitations of such subjects (see the Experimental design and TROUBLESHOOTING sections).

\section{Experimental design}

Experimental details for the TUNL and LD tasks are described below in separate sections. In the first section, some general principles are discussed. First, it should be noted that the protocols for both the TUNL and LD tasks, as described here, are based on the original publications ${ }^{1,3}$, but they have since been slightly modified as a result of ongoing method and task development ${ }^{15}$. For example, in both tasks, the number and dimensions of response locations presented here may differ from previously published work. This protocol describes the standard as currently used in our laboratory.

General considerations. With respect to choosing the appropriate task, we propose that the two tasks presented in this paper be used as alternatives rather than as complementary measures to assess changes in location memory and pattern separation. For example, if the priority is to assess working memory, the TUNL task represents a suitable choice. Alternatively, for a study focusing mainly on pattern separation, the LD task provides a more rapidly acquired alternative. In addition, these tasks may be combined with other touchscreen tasks ${ }^{16,18}$ in a flexible battery ${ }^{14}$ to address specific hypotheses and research requirements. For a discussion of optional batteries, see ref. 16 . This approach may be particularly 
appropriate when there are no specific a priori hypotheses regarding the domains of cognition that will be affected by a given manipulation.

With respect to the experimental design, it is important to recognize that the timing of the experimental manipulation determines the exact procedure. Both tasks require initial pretraining, during which animals learn to touch the screen for a reward. This procedure precedes most touchscreen tasks, and experimental details will be briefly described in the following section. However, for an extensive description of pretraining stages, including flowcharts, see ref. 16. For subsequent TUNL and LD task training, we describe four relatively common experimental designs (cases 1-4) that may result in a somewhat different implementation of either the TUNL or LD protocol. In subsequent sections, these cases will be referred to as appropriate. In case 1, the subject receives treatment before the onset of the experiment (e.g., transgenic models and developmental manipulations). In case 2 , the subject receives treatment before task acquisition but after initial pretraining (e.g., subchronic drug treatment and neurotoxic lesions). In case 3, differences between groups are assessed after animals have reached an asymptotic performance level. This can be done by using a between-subject design after acquisition (e.g., neurotoxic lesion, subchronic drug treatment). Finally, in case 4, a within-subject manipulation after acquisition may be applied, such as in transient systemic drug studies or infusion procedures.

As mentioned, these experimental cases determine how TUNL and LD (and other touchscreen tasks ${ }^{16,18}$ ) are to be implemented. To assess differences in acquisition curves between groups, all animals may be trained continuously (i.e., 5-7 d per week) until performance asymptotes. In this scenario, some animals will be relatively overtrained compared with others, owing to variation in acquisition speed. Even though the TUNL and LD tasks can be used in this way, it may be less useful to assess the acquisition of the learning rule per se, and in both tasks postacquisition probes are likely to be of interest in order to assess delay-dependent performance (TUNL only) or pattern separation-dependent performance (TUNL and LD). If a particular research question is aimed at addressing differences in performance on postacquisition behavioral probes (combined with cases 1 and 2) or in the case of postacquisition experimental manipulations (cases 3 and 4), variation owing to overtraining on the initial acquisition phase, as mentioned above, is not desirable.

Therefore, several options exist as to how (a group of) animals should be advanced through acquisition training as an alternative to continuous training until (beyond) criterion. First, each animal in the group may be trained until it reaches criterion, and then the animals are individually advanced to the manipulation of interest. Although this avoids overtraining and variations in performance level, the group is not synchronized.

Second, a group of animals may all be tested for a prespecified number of acquisition sessions (e.g., on the basis of previous data), and then all animals are advanced to the postacquisition manipulation regardless of the performance level. An advantage is that all animals in the group are synchronized and the manipulation begins for all animals on the same day, which minimizes variability due to external factors and is ideal for pharmacological studies. For example, injections may be administered on the same day(s) for all animals, and decisions concerning the number of days to run a manipulation can be made ad hoc on the basis of the group's mean performance level. This is also particularly important when subjects must be of the same age at the start of each testing phase (for example, in tests of a progressive disease model). However, there will be some variation in the performance levels of the animals at the end of training, and some may not have acquired the initial task to a sufficient baseline level from which to assess alterations in performance due to a manipulation.

We often apply a third option, in which each animal in the group is trained daily (5-7 d per week, as recommended) until it reaches criterion, upon which it is rested without daily training (although food restriction continues). Subjects on rest are usually given one or two reminder training sessions per week unless it is anticipated that all subjects will reach criterion within a few days of each other. If an animal's performance falls below criterion in a reminder session, that animal is trained daily until criterion is reattained. When all animals have reached the criterion (at least) once, they are rebaselined as a group, i.e., all animals are trained daily. Postacquisition manipulations may begin when performance of all subjects has been stable at criterion for at least $2 \mathrm{~d}$. Although subjects receive a different number of training days, precluding plotting of a complete acquisition curve, the animals are synchronized, with minimal variation in their performance levels, and overtraining is minimized.

Pretraining. After the introduction of mild food restriction, the first stage (stage 1) of the protocol is habituation of the animals to the chambers and food rewards (Step 5). In stage 2, the relationship between offset of a visual stimulus on the screen and delivery of reward is introduced. During this stage, a stimulus is presented on the screen, which, in the case of the TUNL and LD tasks, is a white square (or squares). If it is not touched, offset occurs after $30 \mathrm{~s}$ and a reward is delivered, along with illumination of the magazine and a tone (conditioned reinforcer). Touches to stimuli on the screen are encouraged with immediate offset, a triple reward delivery, tone and magazine illumination. When the reward is retrieved, an ITI begins, after which the next trial is automatically initiated. Please note that pretraining for the TUNL and LD tasks differs during this stage with respect to the number of response windows active (Step 6).

Stage 3 is similar to stage 2, but stimulus offset is dependent on the subject touching it. A stimulus is presented, and remains there until it is touched, upon which the stimulus disappears and a reward is delivered accompanied by a tone and magazine illumination. When the animal retrieves the reward and exits the magazine, the ITI begins, after which the next trial begins automatically. Please note that pretraining for the TUNL and LD tasks differs during this stage with respect to the number of response windows active (Step 7A,7B).

Stage 4 is similar to stage 3 , but subjects are required to trigger stimulus presentation, referred to as trial initiation. The session begins with a free reward delivery and magazine illumination, indicating that a trial may be initiated by magazine entry. When an animal nose pokes into the magazine, the magazine light is extinguished and a click sounds, and when the animal withdraws from the magazine stimuli are presented on the screen. Initiation is also required after each ITI (Step 8). 
Figure 1 | Flowchart overview of the main features of the touchscreen TUNL task. (a) The animal initiates a trial by a nose poke into the magazine. The program pseudorandomly selects a trial type and presents one sample stimulus on the screen. The rat is required to touch the sample, after which the delay starts. At the end of the delay, a choice between the previously illuminated sample location and a novel choice location is presented. If the animal responds correctly (touches the choice location), it is rewarded. If it responds incorrectly (touches the sample location), it is punished with a timeout ( $5 \mathrm{~s}$ ). Either response is further followed by an ITI of $20 \mathrm{~s}$, after which the animal is required to initiate the next trial. If the previous response was incorrect, the same sample and choice locations will be presented. This loop will continue until a correct response has been made. The labels in italics indicate steps in which the animal is required to perform an action. (b) As an example (please refer to the text a



b

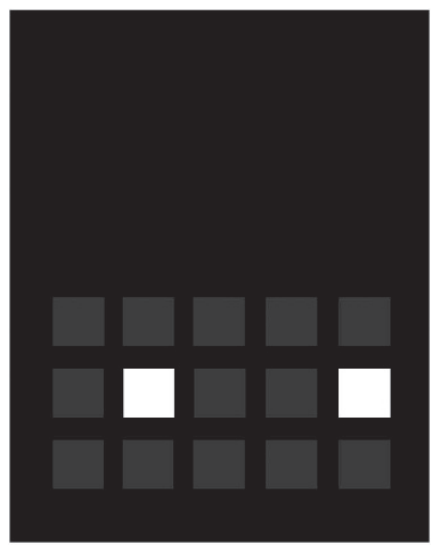

for actual dimensions), the TUNL mask with 15 locations is depicted. The choice phase is shown with two response windows illuminated at 'separation 2 ' (separation is defined as the number of response windows separating two active locations).

Stage 5 is similar to stage 4, but subjects are discouraged from touching blank response windows during stimulus presentation, with stimulus removal and a time-out period in which the house light is inverted. After the time-out, an ITI begins, after which the next trial can be initiated. However, in the pretraining preceding the tasks in this paper, a correction trial in which the same stimulus location is presented is given instead of a new trial. This stage also serves to introduce the subject to the cue signaling incorrect responses (the time-out). By the end of pretraining, subjects should be completing a sufficient number of trials per session (as specified in Step 9) in order to promote completion of sessions in the subsequent task.

The TUNL task. The trial structure of the TUNL task is depicted in a flowchart (Fig. 1). Each session starts with a free food pellet delivered into the food magazine, coinciding with illumination of the magazine. When the animal nose pokes into the magazine to collect the pellet, the magazine light is extinguished, a click sounds (0.2 s) and the first trial is initiated. A sample location (i.e., a white square, see Equipment Setup) is presented the moment the animal exits the magazine. During the sample phase, the animal is required to respond by touching the sample location. Upon touching, the stimulus disappears and the delay starts. One in three sample touches is rewarded with a pellet, and the delivery coincides with a tone and illumination of the magazine light, which extinguishes upon reward collection. At the end of the delay, the magazine light is turned on to indicate that the choice phase can be initiated. As soon as the animal exits the magazine after choicephase initiation, the incorrect sample location and a different correct choice location are simultaneously presented on the screen. Depending on the choice of animals, two scenarios are possible. In the case of a correct response to the novel location, touching the screen leads to reward delivery accompanied by a tone (standard: $1 \mathrm{~s}, 3 \mathrm{kHz}$ ); both stimuli are removed from the screen, and the magazine light is turned on. Upon subsequent magazine entry during reward collection, the magazine light is extinguished and the ITI (20 s) is started. At the end of the ITI, the magazine light is turned on, signaling that the animal can initiate a new trial (i.e., a newly selected distance, sample location and choice location). However, in the case of an incorrect response during the choice phase (a touch to the sample location), the animal is presented with a time-out ( $5 \mathrm{~s}$ ) signaled by house light illumination. Please note that our current standard procedure is to have the house light off during stimulus presentation and ITIs (and on for time-out periods). However, published work on the TUNL task ${ }^{3,60}$ has been performed with house light settings inverted, and we do not have conclusive evidence that these variations affect task performance. After the time-out, the ITI is started, at the end of which the animal can initiate a correction trial (i.e., presentation of the same sample location and choice location). There is typically no limit to the number of correction trials, and further correction trials are presented until a correct response is given. Correction trials are implemented to minimize the formation of biases toward certain locations. After an incorrect response, subsequent correction trials are not added to the total trial number and are not used when calculating percentage correct.

We recommend that initial acquisition of the TUNL task be performed under constant, relatively short delay conditions (e.g., $2 \mathrm{~s}$ ). To further investigate the nature of potential changes in working memory, specific postacquisition probe sessions can be implemented with variation in delay, ITI and separation. Trials during probe sessions are the same as those in regular sessions, with the exception that particular delay conditions or separation conditions (or combinations thereof) are used. Several options can be considered when designing probe sessions. First, it is possible to test animals on sessions in which the combination of delay (short, e.g., $0 \mathrm{~s}$, or long, e.g., $6 \mathrm{~s}$ ) and separation (small or large) is fixed within each session. We recommend testing all four possible permutations, i.e., short-small, short-large, long-small and longlarge. It should be noted that in our experience, animals perform 
Figure 2 | Flowchart overview of the intermediate training and performance probe phases of the location discrimination (LD) task. (a) After initiation, two stimuli are simultaneously presented: one is designated correct and the other incorrect. If a nose poke response to the stimulus in the correct location is made, a reward is delivered. A nose poke response to the incorrect stimulus results in a time-out ( $5 \mathrm{~s}$ ). Either response is further followed by an ITI of $10 \mathrm{~s}$, after which the animal is required to initiate the next trial. If an animal makes seven correct responses in eight consecutive trials, the reward-

location contingency is reversed and trials are repeated until the same level of performance is achieved. The labels in italics indicate steps in which the animal is required to perform an action. (b) As an example (please refer to the text for actual dimensions), the LD mask for mice is shown during the intermediate training phase (see Step 11B(ii)). near chance on the most difficult condition (6-s delay; small separation). Therefore, in studies that assess impairments, this condition could be omitted or adapted to avoid a floor effect.

Second, probe sessions can be designed that alternate selected distances (e.g., small, medium and large) and delays (e.g., 3, 6 and $9 \mathrm{~s}$ ) within a session to assess whether animals perform differently under these varying conditions of task demand (C.A.O., unpublished data).

Third, recent work indicates that, by minimizing both the delay and the ITI, conditions of increased proactive interference can be created, which may be particularly interesting in models of prefrontal pathology, as prefrontal cortex lesions impaired performance on this condition ${ }^{60}$. In this particular study, increased interference was measured in sessions in which the separation was fixed at maximum distance.

The LD task and reversal. During LD training (Fig. 2), each session starts with a free food pellet delivered (or manually placed beforehand) into the food magazine, coinciding with illumination of the magazine. When the animal nose pokes into the magazine to collect the pellet, the magazine light is extinguished, a click sounds $(0.2 \mathrm{~s})$, the first trial is initiated and the stimuli appear on the screen. On each trial, animals are presented with two illuminated locations. Initial acquisition of LD is performed by using a so-called 'intermediate separation' condition, in which response windows 2 and 5 (in the LD task in rats, with seven locations, the latter is window 6) are illuminated (if window 1 is at the extreme left and window 6 is at the extreme right). One location is designated correct $\left(\mathrm{CS}^{+}\right)$and a nose poke to this location results in reward delivery accompanied by a tone (standard: $1 \mathrm{~s}, 3 \mathrm{kHz}$ ) and magazine illumination. Upon reward collection, as the animal enters the magazine, the magazine light is extinguished. Alternatively, a nose poke response to the other (incorrect) illuminated location results in a time-out period (standard: $5 \mathrm{~s}$ ) indicated by house light illumination. Please note that our current standard procedure is to have the house light off during stimulus presentation and ITIs (and on for time-out periods). However, published work on LD has been performed with house light settings inverted, and we do not have conclusive evidence that these variations affect task performance. Stimuli are removed from the screen immediately after either type of response. After magazine activation during reward collection or at the end of the time-out period, an ITI (10 s) commences, after which the magazine is illuminated to indicate that the next trial can be initiated. The animal must continue responding until seven correct responses have been made in eight consecutive trials, which constitutes the acquisition criterion for this task. At this point, the reward contingency is reversed, with the correct location becoming incorrect and vice versa. The animal must then acquire this reversed contingency as demonstrated by again performing seven correct responses in eight consecutive trials. This intermediate training phase is continued until the animal is able to attain the initial location-reward contingency, as well as the subsequent reversal within one session (fixed number of trials per $60 \mathrm{~min}$ ), in three out of four consecutive sessions. The initial correct or incorrect response window designation is counterbalanced across animals. The window designation is also maintained across training sessions (i.e., if window 2 was correct for a particular animal at the end of a training session, it is used as the correct location at the beginning of the subsequent session for that animal).

After completion of the intermediate training phase, the $\mathrm{LD}$ performance probe sessions are conducted. The trial structure of these sessions is identical to the intermediate training phase, except that the animal is either presented with response windows 1 and 6 (rat, 7) illuminated (maximal interstimulus distance; the large separation condition) or windows 3 and 4 (rat, 5) illuminated (minimal interstimulus distance; the small separation condition). Subjects receive an unlimited number of trials per session, with the session terminating after $60 \mathrm{~min}$ or after an animal is able to attain the initial location-reward contingency and subsequent reversal, whichever comes first. All presentations in a given session are either all large or all small, and the two session types are counterbalanced within animal groups. Each animal receives two consecutive sessions of the same probe type, and then, irrespective of performance, receives two consecutive sessions of the other probe type. This alternating paired pattern is repeated for multiple sessions (e.g., 20). Performance on the intermediate training phase may be assessed from the number of sessions or trials required to reach the overall criterion (acquisition and reversal within one session, on three out of four sessions $)^{71}$. The primary measure of probe performance is the average number of trials required to attain acquisition criterion for each probe separation (large and small) $28,71,72$; see the 'Data analysis' sections in the PROCEDURE. 


\section{MATERIALS}

\section{REAGENTS}

- Rats or mice (see Reagent Setup)! CAUTION All experiments using live animals must be approved by and performed in accordance with all relevant governmental and institutional bodies.

- Animal housing (see Reagent Setup)

- Husbandry: rodent food pellets (e.g., rodent pellets, Special Diets Services)

- Reward: we use solid (e.g., Bio-Serv purified rodent dustless precision pellets, $45 \mathrm{mg}$ (rat) or $14 \mathrm{mg}$ (mouse), through Sandown Scientific) or liquid (Yazoo strawberry milkshake, FrieslandCampina) food rewards I CAUTION When you fill the reward dispenser with dustless precision pellets, take care to discard any dust, as this can potentially clog dispensers.

- Cleaning materials (e.g., TriGene, 70\% (vol/vol) ethanol solution, stiff brush)

\section{EQUIPMENT}

- Sound- and light-attenuating box with ventilation system, enclosing an operant chamber and reward delivery system

- Touchscreen operant chambers (from, e.g., Campden Instruments, Med Associates or other commercial suppliers; or custom-made operant system). Note that these are species-specific. Rodent touchscreen operant chambers made by different companies may vary, but they share many common features. The specific model used depends on the experimenter's needs and preference. In Equipment Setup we describe mouse and rat chambers from Campden Instruments and our in-house assembled boxes. Both the TUNL and LD tasks, as well as the majority of other tests ${ }^{16,18}$, have been performed in both

- Camera above the chamber, connected to a closed circuit monitor and a digital video recording device, to monitor and record the animals' behavior (optional but recommended)

- Controlling software and devices; generally available from the operant chamber supplier

- Black plastic masks with response windows (the number and size of which differ between tasks and species; see Equipment Setup)

- Shelves for rat chambers (for some tasks; see Equipment Setup)

- Appropriate data analysis software

- Personal protective equipment (PPE; e.g., disposable medical gloves, lab coat or coveralls, FFP2 mask). $\triangle$ CRITICAL To minimize allergen exposure, PPE should always be worn when you are handling or working near animals.

\section{REAGENT SETUP}

Rats or mice Laboratory-bred or commercially available rats or mice are generally used for testing. There are some advantages to testing male rats and mice, such as avoiding potential estrus cycle-related performance variability in females and potentially increased inter-male aggression when males must be tested in the same apparatus as females. Most commonly for touchscreen operant chamber tests, male Lister hooded rats and male mice on the C57BL/6 or 129 substrain genetic backgrounds have been used. For the LD task, female ${ }^{28}$ and aged ${ }^{71}$ mice have been tested in addition to young male rats and mice. $\Delta$ CRITICAL The TUNL task, as mentioned earlier, can be acquired by rats but not mice. A version for mice is currently under development. ! CAUTION All experiments using live animals must be approved by and performed in accordance with all relevant governmental and institutional bodies. ! CAUTION If animals are not fully grown when food restriction begins, they must be allowed to gain sufficient weight as they continue to grow. For guidance, standard strain growth curves are usually available from the supplier (e.g., http://jaxmice.jax.org/support/weight/index.html).

Animal housing Rats and mice should be housed in groups of $2-5$ animals, with sawdust, bedding and (optional, although recommended) shelter, with cages cleaned regularly. The housing room should be maintained at a constant temperature $\left(21 \pm 2{ }^{\circ} \mathrm{C}\right)$ and humidity $(55 \pm 10 \%)$. Lighting is usually on a 12-h light-dark cycle, and we favor testing rats and mice in the active period of their circadian cycle, as this may enhance activity and potentially learning and memory ${ }^{73-75}$. We advise that researchers consider light-phase conditions, as it can potentially interact with sex, strain, experimental manipulations and so on to influence performance. If you shift or invert the light cycle, allow sufficient time for rats and mice to habituate before commencing behavioral testing (e.g., see ref. 76). We tend to allow $1 \mathrm{~d}$ per hour of shift.
Rewards Two types of reward are typically used: liquid or solid (see MATERIALS). Pellets seem to work well for rats. We use either liquid or solid for mice; liquid rewards may be a better choice in some cases (for mice in particular), e.g., when you are using manipulations that result in motoric changes that could affect chewing, cause dry mouth or reduce motivation. Introduce rewards (pellets or milkshake) inside the home cage to habituate the animals for $1-3 \mathrm{~d}$ before the start of testing. Solid rewards may be scattered on the cage floor; liquid rewards should be put into a shallow, wide-based dish.

\section{EQUIPMENT SETUP}

Campden operant chambers for rats and mice Housed inside a dense fiberboard box, these chambers are equipped with a fan, touchscreen monitor (rat: 15.0 inch, screen resolution 1,024 $\times 768$ (rotated); mouse: 12.1 inch, screen resolution $600 \times 800$ ), tone and click generator, house light (LED), magazine unit (with a light and an IR beam to detect entries; in the standard configuration this is outside the testing arena, on the wall opposite the touchscreen) and pellet dispenser and/or pump connected to bottles of liquid reward. The chambers have a trapezoidal shape (in $\mathrm{cm}$, rat: 30 high $\times$ 33 long (screen-magazine) $\times 25$ wide (at screen) or 13 wide (at magazine); mouse 20 high $\times 18$ long $\times 24$ or 6 wide), composed of three black plastic walls opening onto the touchscreen, intended to help focus the animal's attention to the touchscreen and reward delivery area. The touchscreen uses IR photocells, and therefore it does not require the subject to exert any pressure in order for responses to be registered. Our experience is that rats and mice work most readily and learn fastest with these IR beams, and not when they have to exert any pressure on the screen, although we have not performed a properly controlled experiment to test this. We typically observe rats and mice responding to the screen with their noses (see ref. 16). Access to the chamber is through a transparent lid, which can be secured to the trapezoidal walls with latches during animal testing. The floor is perforated stainless steel raised above a tray lined with filter paper. Two additional photobeams extend between the side walls of the arena, parallel to the screen, to detect the movement of an animal in the front (rat: $\sim 6 \mathrm{~cm}$ from the screen; mouse: $\sim 7 \mathrm{~cm}$ ) or the rear (rat: $\sim 5 \mathrm{~cm}$ from the magazine; mouse: $\sim 3.5 \mathrm{~cm}$ ) parts of the arena. A small IR camera can be installed above the chamber to monitor animals' behavior (optional, but recommended). In Campden rat chambers, a spring-hinged shelf $(24$ wide $\times 6$ long $\mathrm{cm}$ ) can be attached $15 \mathrm{~cm}$ above the floor at a $90^{\circ}$ angle to the screen and mask. In general, attaching a shelf to the mask may reduce impulsive responding and may improve attention directed to stimuli; we have found that this specifically occurs in rats, as this forces rats to rear up before making a choice ${ }^{77}$. Until recently, a shelf has been used in rat LD and TUNL studies $1,3,60$. However, recent task development in our laboratory has shown that rats acquire the TUNL task markedly faster when locations are presented on the lower half of the screen without the use of a shelf (C.A.O., unpublished data), which we therefore recommend in this protocol. We continue to recommend the use of a shelf for the LD task.

Our in-house chambers Housed inside a melamine box, the chambers (modified in our lab from Med Associates operant chambers) are equipped with a fan, an IR touchscreen monitor (in $\mathrm{cm}$, rat: 29.0 high $\times 23.0$ wide; mouse: 16.0 high $\times 21.2$ wide; Craft Data), tone generator, click generator, house light $(\sim 3 \mathrm{~W})$, magazine and pellet dispenser. The touchscreen does not require the subject to exert any pressure in order for touches to be registered. The chambers have a rectangular shape, consisting of a metal frame with clear Perspex walls (in cm, rat: 29 high $\times 31 \mathrm{~cm}$ long $\times 24$ wide; mouse: 13 high $\times 25$ long $\times 19$ wide; excluding space below the floor). Access is through a hinged side wall, secured with a latch during testing. The floor consists of stainless steel bars spaced $1 \mathrm{~cm}$ apart, above a tray lined with filter paper. The magazine is equipped with a light and a photocell nose poke detector. A spring-hinged shelf (in $\mathrm{cm}, 20.5$ wide $\times 6$ long) can be fitted in these rat chambers, $14.0 \mathrm{~cm}$ above the floor, at a $90^{\circ}$ angle to the screen and mask.

Mask and stimulus dimensions: In both the LD and TUNL tasks, animals are trained to touch white (rectangular or square) stimuli on the screen, which serve as response locations. A black plastic mask (in $\mathrm{cm}$, rat in-house: 
38.7 high $\times 30.0$ wide; rat Campden: 35.8 high $\times 28.0$ wide; mouse in-house: 11.8 high $\times 22.8$ wide; mouse Campden: 24.3 high $\times 28.0$ wide) with windows that delineate the response locations is fitted in front of the touchscreen to reduce accidental screen touches and make response locations clearly identifiable from the background. In the LD and TUNL tasks, the size of the (white, square or rectangular) stimuli presented on the screen is similar to the size of the response windows in the mask. Please note that, particularly for LD, dimensions may vary among box types and species.

For the LD task in mice, we recommend using a single row of six response locations located on the bottom half of the screen (no shelf). Response window and stimuli dimensions are as follows: $3 \mathrm{~cm}$ wide $\times 2 \mathrm{~cm}$ high (Campden boxes); or 2.5 wide $\times 2.5$ high (in-house boxes); spaced apart by $1 \mathrm{~cm}$ (Campden boxes) or $0.5 \mathrm{~cm}$ (in-house boxes); and located $2 \mathrm{~cm}$ (Campden boxes) or $1.5 \mathrm{~cm}$ (in-house boxes) above the floor of the chamber. Variation in separation is accomplished by using locations 1 and 6 (large separation), locations 2 and 5 (intermediate separation) or locations 3 and 4 (small separation).

For LD in rats, the data thus far ${ }^{1}$ were collected by using a single row of seven response locations ( $2 \mathrm{~cm}$ wide $\times 2 \mathrm{~cm}$ high, in-house boxes), spaced apart by $1 \mathrm{~cm}$. Response windows were located $1.5 \mathrm{~cm}$ above a hinged shelf, and $16.5 \mathrm{~cm}$ above the floor of the chamber. Variation in separation is accomplished by using locations 1 and 7 (large separation), locations 2 and 6 (intermediate separation) or locations 3 and 5 (small separation).

For the TUNL task (only rats), various response location dimensions and positions have been used ${ }^{3,60}$. We recommend using a mask in which response locations are organized in three rows of five locations $(3.3 \mathrm{~cm}$ wide $\times 3.3 \mathrm{~cm}$ high), spaced apart by $1.5 \mathrm{~cm}$, positioned on the bottom half of the screen (bottom row $1.5 \mathrm{~cm}$ from the grid floor), without the use of a spring-hinged shelf.

Controlling software and devices Controlling software can be purchased from the suppliers of the operant chambers, e.g. Whisker ${ }^{78}$ or ELO software (ELO Touchsystems). Multiple chambers may be controlled by a single computer, although it is important to check that minimum system requirements are met (e.g., memory and graphics cards) to prevent delays in stimuli presentation and chamber responses. All task software is derived from earlier publications and is available (excluding, in some cases, recent modifications) from Campden Instruments or from Med Associates (K-Limbic) or other suppliers. Alternatively, software may be programmed by using common programming languages such as Visual Basic 6.0 (Microsoft).

\section{PROCEDURE}

\section{Preparation for pretraining}

1| If animals are obtained from an outside source (i.e., different from the animal facility in which behavioral testing will occur), allow them to acclimatize after transport without any procedures, with food and water ad libitum, for a minimum of $7 \mathrm{~d}$. Begin handling and weighing the animals after $2 \mathrm{~d}$ of acclimatization.

$\Delta$ CRITICAL STEP Regarding matters such as food restriction and housing, we advise consulting with your institutional animal care regulatory body when you are planning and designing experiments.

$\Delta$ CRITICAL STEP In general, considerations such as group size should, ideally, be based on previous work using comparable animals (i.e., species, strain, age and background) and on the type of behavioral assay.

$\Delta$ CRITICAL STEP If subjects have previously been tested on another instrumental touchscreen task, maintain food restriction and start at pretraining Step 9. As discussed in the Experimental design section, touchscreen tasks (e.g., Step 11A, $11 \mathrm{~B}$; also see refs. 16,18) may be used in flexible combinations and orders by using a battery approach.

2| Weigh each animal for 3 consecutive days with ad libitum food and water, and then calculate the mean free-feeding weight of each animal.

$\triangle$ CRITICAL STEP Ensure that each animal can be reliably identified.

3| After the 7-d acclimatization period, begin food restriction, ensuring that you adhere to institutional and governmental animal care guidelines. Slowly reduce (e.g., over 3-7 d) the weight of individual animals down to the goal weight, which will be a percentage of the measured free-feeding weight (e.g., we use 85-95\%), by controlling the daily amount of food they are given (e.g., for rats, $\sim 7 \mathrm{~g}$ food per $100 \mathrm{~g}$ body weight; for mice, $\sim 2-3 \mathrm{~g}$ food per 25-35 g mouse). Start Step 4 when the animals are close to their goal weights.

$\triangle$ CRITICAL STEP Maintain food restriction throughout touchscreen testing.

$\triangle$ CRITICAL STEP It is important to check the weight of animals daily (mice) or twice a week (rats) throughout the experiment; in our experience, this is particularly important for performance in mice. This also helps habituate the animals to being handled. Aim to avoid weight reduction of $>5 \%$ per day and weight reduction to below $85 \%$ of that observed during free feeding.

4| Introduce reward (pellets or milkshake) inside the cage to habituate the animals for 1-3 d. Solid rewards may be scattered on the cage floor; liquid rewards should be put into a shallow, wide-based dish.

\section{Pretraining}

5| Habituation (stage 1). Start pretraining with a habituation stage; for this, set up the apparatus (see MATERIALS) as appropriate for the planned task (i.e., LD or TUNL) by using the corresponding mask and, optionally, a spring-hinged shelf (as recommended here, only for LD in rats). Turn on all electronic components so that subjects can habituate to them. Place ten reward pellets or $0.2 \mathrm{ml}$ of liquid reward in the reward magazine. Place each rodent into its assigned chamber for $30 \mathrm{~min}$. No task-related software is active during habituation, but when it is available (e.g., Campden Instruments boxes) we 
recommend recording baseline activity. Remove the animal from the chamber and return it to its home cage. Check whether rewards are consumed at the end of the session. Test all subjects on this habituation stage for a minimum of two sessions. The criterion for advancing animals to the next stage of pretraining is consuming all rewards in one session.

$\Delta$ CRITICAL STEP Operant chambers should be cleaned regularly (e.g., once a week or more) to avoid context change during sensitive task phases, to ensure that the touchscreen and IR photobeams retain maximum sensitivity and to prevent accumulation of dirt and excrement. We typically dismantle inner chambers and clean them with surface disinfectants (e.g., TriGene and $70 \%$ (vol/vol) ethanol) and paper towels or a stiff brush.

$\Delta$ CRITICAL STEP Animals require fewer standard rodent food pellets when receiving rewards during training; adjust the daily food allowance as appropriate to maintain goal weights.

$\triangle$ CRITICAL STEP Aim to train, weigh and feed each animal at approximately the same time each day, and use the same operant box for each animal during training. Always counterbalance chambers and testing times across experimental groups. We recommend training animals for one session per day, 5-7 d per week.

$\Delta$ CRITICAL STEP Advance individual subjects to the next pretraining stage when they reach criterion, even if some animals in the group remain at the previous stage(s).

6| Training to associate stimuli on the screen with a reward (stage 2). Set up the apparatus as detailed in MATERIALS, with choice of mask and shelf suitable for the planned behavioral task, and use the software program for this stage with settings as detailed in Experimental design. Place each subject in its assigned chamber and start the session. The session finishes after 60 min or after 100 trials (rat) or 30 trials (mouse) are completed, whichever comes first. After session termination, return each animal to its respective home cage. Advance individual subjects to the next training phase when they achieve a criterion of completing all trials (mice) or 60 trials (rats) within 60 min.

$\triangle$ CRITICAL STEP Please note that rats are typically given the opportunity to complete more trials per session than mice, e.g., 100 as opposed to 30 during pretraining. Rats readily complete a greater number of trials per session than mice, perhaps because the mouse:rat body mass ratio is smaller than the mouse:rat reward pellet size ratio (14 mg:45 mg).

$\triangle$ CRITICAL STEP Please note that pretraining stage 2 is different for the LD and TUNL tasks with respect to the number of response windows active on any given trial. In the LD task, a single response window is illuminated (and responsive to touches) throughout all pretraining stages. In the TUNL task, we recommend having all 15 locations illuminated simultaneously during stage 2 (Step 6) and, initially, during stage 3 (Step 7) of pretraining in order to facilitate acquisition. During stage 3 of TUNL pretraining, the size of the location is scaled down to a single response window.

$\triangle$ CRITICAL STEP At the end of each session, record all crucial data for each subject, such as the number of correct responses and the number of trials completed. Most software programs will record many other measures (see Experimental design).

$\triangle$ CRITICAL STEP If you are testing the effects of a manipulation conducted before the onset of the experiment (case 1 , see Experimental design), ensure that the animals in the experimental and control groups complete comparable numbers of trials per session from stage 3 (Step 7) onward. Cap the number of trials given per session to accommodate the lowest responders.

7| Training to touch stimuli on the screen for a reward (stage 3). Follow option A for LD pretraining and option B for TUNL pretraining.

\section{(A) For LD pretraining}

(i) Repeat the procedure as detailed in Step 6 (stage 2), by using the appropriate software program for stage 3 (see Experimental design). When the animals have reached criterion on stage 3, transfer the animals to stage 4.

\section{(B) For TUNL pretraining}

(i) Repeat the procedure as detailed in Step 6 (stage 2), by using the appropriate software program for stage 3 (see Experimental design). Start this stage by subjecting animals to this task with all 15 response windows activated simultaneously until criterion is reached.

(ii) Next, repeat stage 3, but use an adapted version of the task in which a smaller response location is used that consists of four $(2 \times 2)$ adjacent locations instead of all 15 windows. The location of this square on the screen should be selected pseudorandomly across trials.

(iii) After animals have reached criterion, repeat stage 3 a third time; use a single window as the active response location. Proceed to stage 4 when the animals have reached criterion.

8| Training to initiate trials (stage 4). Repeat the procedure as detailed in Step 6 (stage 2), by using the appropriate software program for stage 4 (see Experimental design). For this stage, ensure that at the start of each session subjects are provided with a single free reward to encourage initiation of the first trial.

9| Punishment for incorrect responses (stage 5). Repeat the procedure as detailed in Step 8 (stage 4), by using the appropriate software program for stage 5 (see Experimental design). The criterion for this stage is completing all trials with 
$\geq 80 \%$ correct (not including correction trials) within $60 \mathrm{~min}$ (rat), or with $\geq 75 \%$ correct within $35 \mathrm{~min}$ (mouse), on two consecutive sessions.

$\triangle$ CRITICAL STEP To minimize the formation of biases toward certain locations, it is crucial that pretraining stage 5 is carried out including correction trials.

$\Delta$ CRITICAL STEP There is likely to be variation in the number of days that animals require to complete pretraining. We suggest resting individual animals when they reach criterion early on this final stage of pretraining, with reminder sessions once or twice per week (and continued food restriction) until the entire group has achieved criterion (please refer to Experimental design). Next, rebaseline all subjects so that the entire group can advance to a specific touchscreen task on the same day. This also ensures that subsequent performance differences on the task cannot be attributed to differences in pretraining performance (relevant for case 1 only, see Experimental design).

10| If subjects are scheduled to receive experimental treatments after pretraining but before task acquisition (case 2, see Experimental design), perform these treatments now, making sure to counterbalance control and experimental groups according to the number of sessions required to complete pretraining. Next, rebaseline the subjects on Step 9 (stage 5) before task-specific training.

\section{Task}

11| Proceed to the TUNL task (option A) or to the LD task and reversal (option B).

$\triangle$ CRITICAL STEP We propose the TUNL and LD tasks as alternatives to assess memory for location and pattern separation in the touchscreen. For details on rationale and task differences, please see the INTRODUCTION. As mentioned earlier, mice are not able to reliably acquire TUNL by using the current protocol (in our experience).

\section{(A) TUNL task}

(i) Train subjects on once-daily sessions of the TUNL task, 5-7 d per week. Provide a single free reward at the start of each session (if your program does not do so automatically). Set up the apparatus as detailed for this task in MATERIALS and the software program for this stage with settings as detailed in Experimental design. We recommend using a short (e.g., 2-s), fixed delay for initial acquisition. Place each subject in its assigned chamber, and start the session. The session usually finishes either after $60 \mathrm{~min}$ or after all trials (e.g., 84) are completed, whichever comes first. $\triangle$ CRITICAL STEP Counterbalance chambers and testing times (time of day) across experimental groups. $\triangle$ CRITICAL STEP Given that performance will be at chance at the start of training (resulting in a relatively high number of additional correction trials), initially limit sessions to 42 trials in $60 \mathrm{~min}$. Continue until subjects can complete number of trials this in $30 \mathrm{~min}$, and then advance to 84-trial sessions. Give each subject an even number of these reduced sessions, such that they can be combined into full 84-trial sessions for analysis. If the subject completes fewer trials than required, the missed trials may be added onto the trials required in the next session (if <10). If this situation occurs frequently, consider capping the number of trials per session further, to accommodate the slowest responders. It is important that animals complete sessions such that all animals are exposed, in a counterbalanced manner, to the same number of trials and trial types (this is particularly important in cases 1 and 2). $\Delta$ CRITICAL STEP At the end of each session, record all crucial data for each subject, such as the number of correct responses and the number of trials completed. Most software programs will record many other measures.

(ii) When all animals have reached criterion at least once, proceed to the next step. The criterion during TUNL acquisition can be set to a certain performance level across all trial types (i.e., all separations), or it can be measured for different separation conditions individually. We recommend a criterion of $80 \%$ correct, on 2 consecutive days, on the two largest separation conditions only. These separations include all trials in which response locations are horizontally separated by two or three inactive locations. In our experience, this corresponds to a stable overall performance of $\sim 70-75 \%$ correct. $\triangle$ CRITICAL STEP To determine which animals have reached criterion, it is essential to perform data analysis daily (Step 11A(iv)). See the TROUBLESHOOTING section for solutions if certain subjects are unable to reach criterion.

$\triangle$ CRITICAL STEP Consider, depending on the experiment, how to proceed when individual animals reach criterion. In the TUNL task, probe sessions that include variation in delay and/or separation are often applied, in which case we recommend resting individual animals when they reach criterion (with regular reminder sessions) until all animals have acquired the task (for a discussion of scenarios, see Experimental design).

? TROUBLESHOOTING

(iii) To investigate the effects of postacquisition treatment (case 4, see Experimental design) on task manipulations (i.e., probe sessions) that are not part of regular task acquisition, expose animals to these conditions before treatment to avoid confounds due to novelty or contextual change, as well as to allow for a within-subject pre- and posttreatment comparison of performance level. Expose animals to these probe sessions until performance is stable.

(iv) To investigate the effects of postacquisition treatment (cases 3 or 4, see Experimental design), perform the scheduled experimental treatment now, making sure to counterbalance control and experimental groups according to acquisition 
speed (number of trials to reach criterion) and performance (percentage correct on the final phase of acquisition). For, e.g., microinfusion studies (case 4), animals should be rebaselined after surgery until a stable performance level is reached. Subjecting animals to the relevant probes should also occur at this point. Before commencing subsequent vehicle and drug infusion, a mock infusion involving the insertion of the infusion cannula only should be performed, followed by a vehicle infusion to assess nonspecific effects on performance.

(v) Post-training manipulations: probe sessions. Expose animals to probe sessions, the nature of which may vary depending on the experimental hypothesis (for suggestions, see Experimental design). Postacquisition within-subject treatments (case 4) may be performed at this stage in an appropriately controlled way (e.g., Latin square or crossover design).

$\triangle$ CRITICAL STEP Probe sessions are usually similar to acquisition sessions with respect to the number of trials and maximum session time $(1 \mathrm{~h})$. Ensure that, in each probe session, animals are exposed to the experimental conditions (i.e., trials with certain separation and/or delays) in a counterbalanced manner, depending on the probe design.

$\Delta$ CRITICAL STEP To minimize the development of mediating strategies, alternating different probe sessions is recommended, particularly when you are using probe sessions of a fixed maximum separation. We recommend a maximum of three consecutive sessions.

$\triangle$ CRITICAL STEP The order of exposure to probes should be counterbalanced between groups. Regular analysis of performance is recommended to assess whether sufficient data on all permutations of delays and separations have been collected.

(vi) Data analysis. Record the following behavioral variables for each subject: number of sessions required to complete pretraining (Steps 5-9), and/or individual pretraining stages; accuracy; number of correction trials per session; number of incorrect correction trials per session, to assess perseverative responding to certain locations on the screen; correct response latency (ms), which is the time between exiting the magazine at initiation and making a correct response; incorrect response latency (ms), which is the time between exiting the magazine at initiation and making an incorrect response; reward collection latency (ms), which is the time between making a correct response and entering the magazine to collect the reward; and number of screen touches during the ITI and time out. Accuracy, expressed as the percentage of correct responses, should be calculated as (number of correct responses)/(number of correct responses + number of incorrect responses) $\times 100$. Note that this does not include data from correction trials. Overall accuracy, as well as accuracy on particular separation and delay conditions, should be calculated for each session. This is particularly important during acquisition in order to determine whether animals have reached criterion. Depending on the results, it may be advisable to perform analysis of the above variables on trials only during which the actual (self-imposed) delay does not exceed a certain value.

For analysis, compare variables between experimental groups by using the appropriate statistical tests. Exact choice of parameters and tests will depend on the nature of the experiment (e.g., as described in the different cases in Experimental design) and the number of experimental groups. For cases 1 and 2, we often compare acquisition curves (plotted as percentage correct on daily sessions) by using a repeated-measures ANOVA. Alternatively (especially when animals have been rested after reaching criterion), the number of trials or sessions to criterion can be compared with a $t$ test or ANOVA for independent samples. For cases 3 and 4, a within-subject comparison can be performed by comparing subjects at asymptotic performance levels or on specific probe sessions, before and after experimental manipulation. In all instances, response latencies and reward collection latencies should be analyzed to assess whether results may be explained by nonspecific effects on performance, such as changes in motivation. In addition, statistical tests should be applied after checking for the appropriate assumptions for the test in question (e.g., the normality of the data).

(B) LD task and reversal

(i) LD intermediate training phase. Assign subjects (in each experimental condition, if pre-experimental or preacquisition manipulations have been conducted) to two groups. Groups should be counterbalanced on the basis of the number of sessions required to complete pretraining, and on mean performance on the last $2 \mathrm{~d}$ of pretraining. For one group, the stimulus in the left window (location 2) will be correct in the first intermediate training session, and for the other the right window (location 5 for the mouse-mask; location 6 for the rat-mask, see MATERIALS, Equipment Setup).

(ii) Begin training on once-daily sessions of LD intermediate-separation training, 5-7 d per week. Provide a single free reward (if your program does not do this automatically). Set up the apparatus as detailed for this task in MATERIALS and the software program for this stage with settings as detailed in Experimental design, with reward contingency as appropriate for each subject. Place each subject in its assigned chamber and start the session. The correct location for a particular animal at the end of an intermediate training session is used as the correct location for that animal at the beginning of the next session. Sessions terminate after 60 trials (mice) or 100 trials (rats), or after 60 min, whichever comes first. $\Delta$ CRITICAL STEP Ensure that animals in experimental and control groups complete a comparable numbers of trials per session throughout task acquisition (this is particularly important in cases 1 and 2, see Experimental design). This can be done by only giving subjects 30 (mice) or 50 (rats) trials per session on the first few sessions. Continue with these reduced sessions until subjects can complete them in $30 \mathrm{~min}$. Ensure that you give an even number of such sessions so that they can be combined for data analysis. If a subject completes fewer trials than required, the missed trials may be added to the 
trials required in the next session by increasing the maximum number of trials to be completed (only if less than 10). Alternatively, they must be given in an additional session to ensure that exposure to the task is equal between subjects. If the problem persists, consider further capping the number of trials given per session to accommodate the lowest responders. $\triangle$ CRITICAL STEP At the end of each session, record crucial data for each subject (e.g., number of correct responses, number of trials completed, number of within-session reversals). However, most software programs will record many other measures.

(iii) When all animals have reached the overall criterion, proceed to the next step. The overall criterion for the intermediate training phase is achieving acquisition and reacquisition of the reversed contingencies in three out of four consecutive sessions. Regardless of the experimental manipulation (cases 1-4), we suggest resting animals when they reach criterion, with reminder sessions once or twice per week (and continued food restriction) until the entire group has achieved criterion; see Experimental design. If an animal's performance falls below criterion in a reminder session, that animal is trained daily until criterion is reattained. When all animals have reached the overall criterion once, rebaseline the group (i.e., test all animals daily) before beginning probe sessions.

\section{? TROUBLESHOOTING}

(iv) If subjects are scheduled to receive experimental treatments after intermediate training but before probe sessions (i.e., postacquisition between-subject manipulations; case 3), perform these treatments when all animals have achieved the overall criterion once, making sure to counterbalance control and experimental groups according to acquisition performance. Next, rebaseline all animals. Proceed to Step $11 \mathrm{~B}(\mathrm{v})$ when performance of all subjects has been stable at criterion for at least 2 out of 3 consecutive days.

$\Delta$ CRITICAL STEP To investigate the effects of postacquisition treatments (case 3 or 4 ) on behavioral challenges (i.e., probe sessions), animals should be exposed to these conditions before treatment in order to avoid confounds owing to novelty or contextual change, as well as to allow for a within-subject pre- and post-treatment comparison of performance level. For microinfusion studies, animals should be rebaselined until they are stable before a mock infusion involving the insertion of the infusion cannula only, followed by a vehicle infusion to assess nonspecific effects on performance.

(v) LD task performance probe sessions. Assign subjects (of each experimental condition, if manipulations have been performed) into four groups, counterbalanced according to the number of days to reach the criterion and the number of reversals to the criterion in the intermediate training phase. When testing the LD task in mice by using a mask with six locations, one group will receive the small separation condition first with the stimulus in the left window correct in the first probe session (location 3), and another will receive the small separation condition with right correct (location 4). The third group will receive the large separation condition with left correct (location 1), whereas the final group will receive the large separation condition with right correct (location 6). In the case of a mask with seven windows, as used in the rat LD task, the middle location (location 4) remains inactive. The small separation condition is represented by locations 3 and 5, whereas the large separation condition is represented by locations 1 and 7 .

(vi) Proceed as in Step 11B(ii), but use a modified software program (see Experimental design). Postacquisition withinsubject treatments (case 4) may be performed at this stage in an appropriately controlled way, e.g., Latin square or crossover design. Train subjects in daily sessions, 6-7 d per week if possible. There is no trial limit, and the session will terminate after $60 \mathrm{~min}$ or when the animal is able to acquire the initial location-reward contingency and the subsequent reversal (whichever occurs first). Give animals two consecutive sessions of each probe type (large, small) for multiple days (e.g., 20). As before, the final correct location of a session is used as the starting correct location in the next session, except when an animal is switched between probe session types, in which case the correct location is designated based on the initial counterbalancing and not the previous session.

(vii) Data analysis. Record or calculate the following behavioral output measures per animal: number of sessions required to complete pretraining (Steps 5-9), and/or individual pretraining stages; sessions/trials to reach criterion in the intermediate training phase; average number of trials required to attain acquisition criterion for each probe separation (small, large) separately; average number of trials required to attain reversal (reacquisition) criterion for each probe separation; correct (or incorrect) reaction time, defined as the average latency to respond to the correct (or incorrect) stimulus, following the presentation of stimuli, averaged over all probe sessions; magazine latency, defined as the latency to enter the magazine to collect reward following a correct response, averaged over all probe sessions; and number of screen touches during the ITI and time-out. The results for the average number of trials required to attain acquisition criterion for each probe separation may be highly variable, and data may be absent for some sessions if the animal did not attain criterion (see the TROUBLESHOOTING section). Thus, for analysis we suggest taking the average of this measure from several sessions, e.g., all sessions of a given separation.

For analysis, compare variables described above between experimental groups by using the appropriate statistical tests. Exact choice of parameters and tests will depend on the nature of the experiment (e.g., as described in the different cases in Experimental design), and the number of experimental groups. The number of trials or sessions to overall criterion during the intermediate separation training phase can be compared between experimental groups, 
e.g., by using a $t$ test for independent samples. To analyze the average number of trials taken to acquire (or reacquire) the within-session criterion on the separation probes, we typically subject data to a repeated-measures ANOVA with experimental group as a between-subject factor, and separation (small, large) as a within-subject factor. In all instances, response latencies and reward collection latencies should also be analyzed to assess whether results may be explained by nonspecific effects on performance, such as changes in motivation. In addition, statistical tests should be applied after checking for the appropriate assumptions for data distribution.

\section{? TROUBLESHOOTING}

\section{? TROUBLESHOOTING}

For general troubleshooting advice on the touchscreen operant chamber testing method, including technical issues, see Table 1.

TABLE 1 | Troubleshooting table.

\begin{tabular}{|c|c|c|}
\hline Problem & Possible reason & Solution \\
\hline \multirow[t]{2}{*}{$\begin{array}{l}\text { Incomplete consumption } \\
\text { of reward }\end{array}$} & Animal is insufficiently food restricted & Decrease weight as regulations permit \\
\hline & $\begin{array}{l}\text { Animal is insufficiently habituated to } \\
\text { rewards }\end{array}$ & Provide rewards in the home cage for additional days \\
\hline \multirow[t]{5}{*}{ Unstable or poor performance } & Low or excessive motivation & $\begin{array}{l}\text { Pay closer attention to weight control; consider temporary } \\
\text { feeding separation, according to the rate of responding }\end{array}$ \\
\hline & Aversion to mask or touchscreen & $\begin{array}{l}\text { Increase exploration of the mask and screen by applying food } \\
\text { reward on the mask (e.g., peanut butter, pellets or other) }\end{array}$ \\
\hline & Excessive fighting in home cages & $\begin{array}{l}\text { Monitor the home cages and general health of animals, } \\
\text { separate if necessary }\end{array}$ \\
\hline & $\begin{array}{l}\text { Stressors in housing room } \\
\text { (e.g., noise) }\end{array}$ & $\begin{array}{l}\text { Make frequent observations of room and cage, move } \\
\text { if necessary }\end{array}$ \\
\hline & Poor learning ability & Exclusion may be necessary \\
\hline \multirow[t]{4}{*}{$\begin{array}{l}\text { Abrupt decline in performance } \\
\text { and/or trial completion }\end{array}$} & $\begin{array}{l}\text { Touchscreen error (e.g., nonresponsiveness, } \\
\text { not displaying images) }\end{array}$ & $\begin{array}{l}\text { Check physical connections, clean, run test program } \\
\text { (if available), recalibrate, reboot the system }\end{array}$ \\
\hline & Reward delivery ceases or is inconsistent & $\begin{array}{l}\text { Check for physical blockage or disconnection, check for } \\
\text { interface errors, replace the reward dispenser }\end{array}$ \\
\hline & Initiation not detected & $\begin{array}{l}\text { Clean the magazine photobeam, check physical connec- } \\
\text { tions, replace if faulty }\end{array}$ \\
\hline & $\begin{array}{l}\text { Controlling system error } \\
\text { (software or hardware) }\end{array}$ & $\begin{array}{l}\text { Check physical connections, reboot the system and change } \\
\text { hardware if necessary }\end{array}$ \\
\hline $\begin{array}{l}\text { Animal appears to make an } \\
\text { unusually low or high number } \\
\text { of beam crosses (Campden only) }\end{array}$ & IR beam failure & $\begin{array}{l}\text { Clean the IR beam pathway, check the position of the IR } \\
\text { switch, replace faulty beams }\end{array}$ \\
\hline
\end{tabular}

\section{TUNL task-specific troubleshooting}

In TUNL, individual animals may fail to progress through a session. This is usually due to the fact that rats show perseverative responding to certain locations on the screen (location bias), as occasionally found in trials in which both stimuli appear in one column (rats may prefer responding to the lower stimulus). In our experience, the problem is likely to reduce with further training. If this does not occur, the number of sequential correction trials can be capped. In addition, individual animals may have difficulties reaching criterion (especially when a different strain or disease model is used), even though recent changes such as increased stimulus size have produced substantial improvement in the acquisition rate of the TUNL task (e.g., ANTICIPATED RESULTS). We suggest the following solutions in the case of slow learning rates. First, if animals have acquired the nonmatching rule but are not attaining the (relatively stringent) criterion, lowering this criterion may be 
preferable to excluding individuals, because group performance can be compared on subsequent probe sessions. It should be noted that lowering the criterion will probably result in a more variable performance level during further testing (e.g., during probes). This is not desirable in, for example, pharmacological studies (e.g., case 4) in which treatments are often implemented within a single session. As an alternative to lowering the criterion, task parameters can be adjusted to facilitate learning (e.g., omitting smaller separations). However, in this scenario, care should be taken to avoid the use of mediating strategies, and we advise alternating easier sessions with normal sessions in which all separations are presented. Finally, certain animals may have to be excluded from the experiment if they are unable to reach criterion.

\section{LD task-specific troubleshooting}

In the LD task, if the subject does not achieve acquisition and/or reversal (reacquisition) criterion in a given session, data (trials to criterion) will not be generated. If there are several such instances of missing data for a given subject, it may be necessary to exclude the subject from analysis. For example, one might require that data be available from at least one session from each session pair, or that at least five sessions of data be available from a full ten sessions of each separation. If there is a large amount of missing data, the number of mice failing to achieve acquisition and/or reversal (reacquisition) criterion may be analyzed and compared between groups. This problem is likely to be more prevalent with mice, which may demonstrate a more variable performance and complete fewer trials per session. Reversal data are more likely to be incomplete than acquisition, because acquisition precedes reversal in each session. Note that acquisition data may still be analyzed even if reversal data are incomplete, although this may increase variability of acquisition performance.

\section{TIMING}

Approximate timing for each step below is indicated in number of sessions (i.e., days). As a rule, allow up to 80 min per $d$ per animal per testing session from Step 5 onward. These 80 min include 60 min of testing time, plus an additional 20 min for transporting animals from the home cage to the testing room, setting up software and so on. Cumulative time taken to test all animals on a daily basis depends on the capacity to load multiple animals per test run (i.e., number of chambers). Subsequent values for the number of days (sessions) it takes to execute these experiments reflect the approximate time it takes to test an average cohort of animals on each stage of the task and are estimates based on our experience.

Steps 1-4, preparation for pretraining: $\sim 3$ to $10(3+7)$ d. Timing depends on whether animals are acquired from an external source, in which case a 7-d acclimatization period is required before the onset of food restriction. After acclimatization, allow for $\sim 3 \mathrm{~d}$ of initial food restriction before starting stage 1 pretraining. Regular handling and weighing of animals can be started $\sim 2 \mathrm{~d}$ after arrival. Reserve an average time per animal per day of $\sim 5 \mathrm{~min}$.

Steps 5-9, pretraining: 10-30 sessions. For TUNL task pretraining, up to 15 sessions may be needed because of the extended stage 3 (i.e., repeating Step 7B). For LD pretraining in mice, occasionally up to 30 sessions may be expected. Full pretraining is only necessary before the first instrumental task on which an animal is tested; otherwise, expose the animals to Step 9 only, by using the box setup appropriate for the planned task (see MATERIALS).

Step 10, experimental treatment (e.g., surgery): timing of this depends on the procedure, but allow for at least $7 \mathrm{~d}$ of recovery in the case of surgery. Please note that this step is optional and can be implemented in the case of manipulations that are planned after pretraining but prior to task acquisition (i.e., Case 2, see Experimental design). Please note that for the TUNL and LD tasks, we often apply experimental treatment after task acquisition but prior to probe sessions. This has been earlier described as hypothetical options in Experimental design (Cases 3 and 4 ) and is described in the procedure as part of Step 11A(iv) (TUNL) or Step 11B(iii) (LD).

Step $11 \mathrm{~A}, \mathrm{~B}$, training on the TUNL and LD tasks. In general, the duration of training of (any) touchscreen task, with respect to the number of sessions (i.e., days), will depend on experimental design factors such as choice of animals, experimental manipulations (e.g., surgery) and research questions (e.g., use of probes). Here we provide approximate timing with respect to the number of sessions (i.e., days) of several stages.

Step $11 \mathrm{~A}(\mathrm{i}, \mathrm{ii})$, TUNL task acquisition: $\sim 30-35$ sessions are required for animals to reach criterion (using the recommended $3 \times 5$ mask on the lower half of the screen); for details, see ANTICIPATED RESULTS.

Step $11 \mathrm{~A}(\mathrm{iii}-\mathrm{v})$, the number of additional probe sessions required depends on the experimental design and variability in performance. In our experience, 4-10 sessions per probe condition may be needed.

Step 11B(ii,iii), LD acquisition on the intermediate training phase: 20-25 (rats) or 20-40 (mice) sessions may be expected. Step $11 \mathrm{~B}(\mathrm{vi})$, probing in LD on small-versus-large separation conditions may require an additional 20 sessions (rats and mice), again depending on the experimental setup and variability.

\section{ANTICIPATED RESULTS The TUNL task}

The TUNL task is particularly interesting for assessing differences in delay-dependent and separation-dependent performance between groups, such as those measured during postacquisition probes. The example data described in this section were 
Figure 3 | Average performance of male Lister hooded rats on TUNL probe sessions for different delays and separations. Performance during short delay condition ( $0.5 \mathrm{~s}$, dashed line) is higher than that during long delay conditions (9 s, solid line). In addition, within each delay condition, accuracy is dependent on the spatial separation, which is expressed as the distance (i.e., number of blank windows) between response locations. Error bars show s.e.m.; $n=12$. A significant effect of separation $(P<0.001)$, delay $(P<0.001)$ and a significant interaction $(P=0.005)$ was found.

collected in healthy 10-month-old male Lister hooded rats $(n=12)$, and averages are presented \pm s.e.m. The mask dimensions specified in this protocol were used (see MATERIALS, $3 \times 5$ design, positioned on the lower half of the screen), and in this particular experiment the largest separation was omitted during initial acquisition (i.e., trials on which the far left and far right column are lit were not used; please note that we currently recommend including the largest separation during acquisition). Throughout task acquisition, a relatively short delay (2 s) was used. Animals were subjected to task acquisition until criterion was reached, after which individual rats were rested and rebaselined twice a week (see Experimental design). The task was acquired by all 12 rats used in this study, in an average of 1,939 \pm 79 trials or $34 \pm 1$ daily sessions (C.A.0., unpublished data). This is based on the criterion (as described in Step 11A(ii)) of $80 \%$ correct, on 2 consecutive days on the largest separation in this task setup (i.e., trial types where lit response locations are separated, horizontally, by two blank squares). The overall performance level during rebaseline sessions after all rats had reached the criterion was $72.7 \pm 1.1 \%$.

To measure delay-dependent and separation-dependent performance, ten probe sessions with different delay and separation conditions (within session) were implemented. During probe sessions, all possible combinations of sample and choice location were presented, including the largest possible separation (i.e., three inactive response locations between the sample and choice location). The average performance on these probe sessions was calculated for different trial types with respect to delay and separation. Trial types were collapsed into four separation categories ranging from maximum (three squares) to adjacent (zero squares) separation (i.e., separation is the number of response windows between the two active locations, horizontally). In this experiment, the mean correct response latency was $3.28 \pm 0.31 \mathrm{~s}$ and the incorrect response latency was $3.96 \pm 0.38 \mathrm{~s}$. Average time to collect the reward after a correct response was $1.22 \pm 0.15 \mathrm{~s}$. As can be seen from the graph (Fig. 3), performance depends on both separation and delay, as there is a significant within-subject effect of separation $(P<0.001)$, delay $(P<0.001)$ and an interaction $(P=0.005)$ between the two. Expected results on separation and delay in animal models of, for example, prefrontal cortex or hippocampus pathology, remain to be determined. It has been shown that the prefrontal cortex is necessary for delay-dependent performance in the TUNL task ${ }^{60}$, and that a working memory deficit of up to $15 \%$ may be expected on longer delays $(6 \mathrm{~s})$ without changes in performance during no-delay conditions or when using similar separations ${ }^{60}$. In addition, work in animals with hippocampal lesions showed a similar drop in performance (up to $20 \%$ ) on long (but not short) delay conditions (6 s), and animals were impaired on small, but not large, separations (impairment of $\sim 10 \%$; ref. 3 ).

\section{Location discrimination}

As an example of LD task performance, the effect of global Tnik (Traf2 and NcK interacting kinase) gene knockout in male C57BL/6 $\times 129 S 5$ mice (ref. 72) is shown (Fig. 4) compared with control mice of the same background. Task acquisition in the intermediate training phase was not different between groups (wild-type $458.1 \pm 45.8$; Tnik-/- $576.3 \pm 74.2$ trials to criterion, $P=0.248$ ). However, this genetic manipulation impairs task performance in a separation-dependent manner on probe sessions; performance is only impaired in the probe session with minimum distance between stimulus locations (small separation). There were no effects of genotype on response latencies (large separation: wild-type $5.0 \pm 1.1 \mathrm{~s}$; Tnik-/$5.2 \pm 0.8 \mathrm{~s}$; small separation: wild-type $4.9 \pm 0.7 \mathrm{~s} ;$ Tnik $^{-/}-5.0 \pm 0.9 \mathrm{~s}$.). For this analysis, the average number of trials needed for animals to reach criterion (seven correct touches out of eight responses for acquisition and reversal) was

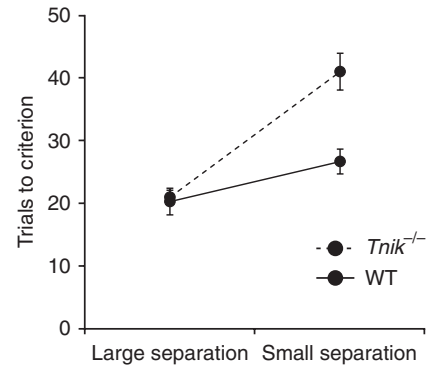

Figure 4 | Average performance of Tnik-/- $(n=12$, dashed line) and wild-type (WT) mice of the same background (C57BL/6 $\times 12955, n=8$, solid line) on the LD task. Performance is depicted as the mean number of trials to criterion during both the acquisition and reversal phase, in both small and large separation probe conditions (error bars s.e.m.), genotype $\times$ separation interaction $P<0.01$; small separation $P<0.05$. Between-group differences are selectively observed in the small separation condition, with the Tnik-/- group requiring significantly more trials to reach the performance criterion ${ }^{30}$, redrawn from ref. 72 . 
calculated for each separation condition. Differences between groups were tested by using an independent samples $t$ test or a repeated-measures ANOVA (genotype as a between subject factor; separation as a within-subject factor). A paired samples $t$ test was used for post hoc analysis of significant interaction effects. Manipulations impairing hippocampal function, or specifically reducing hippocampal neurogenesis, have a similar effect ${ }^{28}$. A similar pattern of results is anticipated for rats, in light of the separation-dependent impairment of hippocampus-lesioned animals, found using a previous version of this protocol $^{1}$. Conversely, manipulations increasing neurogenesis may selectively improve performance ${ }^{71}$.

ACKNOWLEDGMENTS The protocols described here are those used in our laboratory at present, and were written by current members of the group. However, many researchers have contributed to the development of touchscreen tasks and we would like to gratefully acknowledge their contribution. They include S. Bartko, J. Brigman, S. Forwood, C. Graybeal, A. Izquierdo, L. Lyon, A. Marti, K. McAllister, S. McTighe, J. Nithianantharajah, C. Romberg, J. Talpos and B. Winters. The research leading to these results has received support from the Innovative Medicine Initiative Joint Undertaking under grant agreement no. 115008, of which resources are composed of a European Federation of Pharmaceutical Industries and Associations in-kind contribution and financial contribution from the European Union's Seventh Framework Programme (FP7/2007-2013); and the Wellcome Trust/Medical Research Council (089703/Z/09/Z) and Alzheimer's Research UK (ART/PG2006/5). A.E.H. receives funding from the European Union Seventh Framework Programme under grant agreement nos. 241995 (Project 'GENCODYS') and 242167 (Project 'SYNSYS').

AUTHOR CONTRIBUTIONS All authors contributed to the writing of this manuscript. C.A.O. coordinated this effort.

COMPETING FINANCIAL INTERESTS The authors declare competing financial interests: details are available in the online version of the paper.

Reprints and permissions information is available online at http://www. nature. com/reprints/index.html.

1. McTighe, S.M., Mar, A.C., Romberg, C., Bussey, T.J. \& Saksida, L.M. A new touchscreen test of pattern separation: effect of hippocampal lesions. Neuroreport 20, 881-885 (2009).

2. Talpos, J.C., Dias, R., Bussey, T.J. \& Saksida, L.M. Hippocampal lesions in rats impair learning and memory for locations on a touch-sensitive computer screen: the 'ASAT' task. Behav. Brain Res. 192, 216-225 (2008).

3. Talpos, J.C., McTighe, S.M., Dias, R., Saksida, L.M. \& Bussey, T.J. Trial-unique, delayed nonmatching-to-location (TUNL): a novel, highly hippocampus-dependent automated touchscreen test of location memory and pattern separation. Neurobiol. Learn. Mem. 94, 341-352 (2010).

4. Talpos, J.C., Winters, B.D., Dias, R., Saksida, L.M. \& Bussey, T.J. A novel touchscreen-automated paired-associate learning (PAL) task sensitive to pharmacological manipulation of the hippocampus: a translational rodent model of cognitive impairments in neurodegenerative disease. Psychopharmacology 205, 157-168 (2009).

5. Morris, R. Developments of a water-maze procedure for studying spatial learning in the rat. J. Neurosci. Methods 11, 47-60 (1984).

6. Barnes, C.A. Memory deficits associated with senescence: a neurophysiological and behavioral study in the rat. J. Comp. Physiol. Psychol. 93, 74-104 (1979).

7. Olton, D.S. The radial arm maze as a tool in behavioral pharmacology. Physiol. Behav. 40, 793-797 (1987).

8. Olton, D.S. Mazes, maps, and memory. Am. Psychol. 34, 583-596 (1979).

9. Dunnett, S.B. Comparative effects of cholinergic drugs and lesions of nucleus basalis or fimbria-fornix on delayed matching in rats. Psychopharmacology 87, 357-363 (1985).

10. Bierley, R.A. \& Kesner, R.P. Short-term memory: the role of the midbrain reticular formation. J. Comp. Physiol. Psychol. 94, 519-529 (1980).

11. Aggleton, J.P., Keith, A.B., Rawlins, J.N., Hunt, P.R. \& Sahgal, A. Removal of the hippocampus and transection of the fornix produce comparable deficits on delayed non-matching to position by rats. Behav. Brain Res. 52, 61-71 (1992).

12. Deadwyler, S.A., Bunn, T. \& Hampson, R.E. Hippocampal ensemble activity during spatial delayed-nonmatch-to-sample performance in rats. J. Neurosci. 16, 354-372 (1996).
13. Hampson, R.E. \& Deadwyler, S.A. Ensemble codes involving hippocampal neurons are at risk during delayed performance tests. Proc. Natl. Acad. Sci. USA 93, 13487-13493 (1996).

14. Bussey, T.J. et al. New translational assays for preclinical modelling of cognition in schizophrenia: the touchscreen testing method for mice and rats. Neuropharmacology 62, 1191-1203 (2012).

15. Bussey, T.J. et al. The touchscreen cognitive testing method for rodents: how to get the best out of your rat. Learn. Mem. 15, 516-523 (2008).

16. Horner, A.E. et al. The touchscreen operant platform for testing learning and memory in rats and mice. Nat. Protoc. 8, 1961-1984 (2013).

17. Nithianantharajah, J. et al. Synaptic scaffold evolution generated components of vertebrate cognitive complexity. Nat. Neurosci. 16, 16-24 (2013).

18. Mar, A. et al. The touchscreen operant platform for testing executive function in rats and mice. Nat. Protoc. 8, 1985-2005 (2013).

19. Wong, A.A. \& Brown, R.E. Visual detection, pattern discrimination and visual acuity in 14 strains of mice. Genes Brain Behav. 5, 389-403 (2006).

20. Prusky, G.T., Harker, K.T., Douglas, R.M. \& Whishaw, I.Q. Variation in visual acuity within pigmented, and between pigmented and albino rat strains. Behav. Brain Res. 136, 339-348 (2002).

21. Treves, A. \& Rolls, E.T. Computational constraints suggest the need for two distinct input systems to the hippocampal CA3 network. Hippocampus 2, 189-199 (1992).

22. Treves, A., Tashiro, A., Witter, M.P. \& Moser, E.I. What is the mammalian dentate gyrus good for? Neuroscience 154, 1155-1172 (2008).

23. Leutgeb, J.K., Leutgeb, S., Moser, M.B. \& Moser, E.I. Pattern separation in the dentate gyrus and CA3 of the hippocampus. Science 315, 961-966 (2007).

24. Gilbert, P.E., Kesner, R.P. \& Lee, I. Dissociating hippocampal subregions: double dissociation between dentate gyrus and CA1. Hippocampus 11, 626-636 (2001).

25. Yassa, M.A. \& Stark, C.E. Pattern separation in the hippocampus. Trends Neurosci. 34, 515-525 (2011).

26. Gilbert, P.E., Kesner, R.P. \& DeCoteau, W.E. Memory for spatial location: role of the hippocampus in mediating spatial pattern separation. J. Neurosci. 18, 804-810 (1998).

27. McHugh, T.J. et al. Dentate gyrus NMDA receptors mediate rapid pattern separation in the hippocampal network. Science 317, 94-99 (2007).

28. Clelland, C.D. et al. A functional role for adult hippocampal neurogenesis in spatial pattern separation. Science 325, 210-213 (2009).

29. DeCarolis, N.A. \& Eisch, A.J. Hippocampal neurogenesis as a target for the treatment of mental illness: a critical evaluation. Neuropharmacology 58, 884-893 (2010).

30. Eisch, A.J. et al. Adult neurogenesis, mental health, and mental illness: hope or hype? J. Neurosci. 28, 11785-11791 (2008).

31. Sahay, A. \& Hen, R. Adult hippocampal neurogenesis in depression. Nat. Neurosci. 10, 1110-1115 (2007).

32. Lucassen, P.J. et al. Regulation of adult neurogenesis by stress, sleep disruption, exercise and inflammation: implications for depression and antidepressant action. Eur. Neuropsychopharmacol. 20, 1-17 (2010).

33. Reif, A. et al. Neural stem cell proliferation is decreased in schizophrenia, but not in depression. Mol. Psychiatry 11, 514-522 (2006).

34. Toro, C.T. \& Deakin, J.F. Adult neurogenesis and schizophrenia: a window on abnormal early brain development? Schizophr. Res. 90, 1-14 (2007).

35. Jin, K. et al. Increased hippocampal neurogenesis in Alzheimer's disease. Proc. Natl. Acad. Sci. USA 101, 343-347 (2004).

36. Donovan, M.H. et al. Decreased adult hippocampal neurogenesis in the PDAPP mouse model of Alzheimer's disease. J. Comp. Neurol. 495, 70-83 (2006). 
37. Wen, P.H. et al. The presenilin-1 familial Alzheimer disease mutant P117L impairs neurogenesis in the hippocampus of adult mice. Exp. Neurol. 188 , 224-237 (2004).

38. Thompson, A., Boekhoorn, K., Van Dam, A.M. \& Lucassen, P.J. Changes in adult neurogenesis in neurodegenerative diseases: cause or consequence? Genes Brain Behav. 7 (suppl. 1), 28-42 (2008).

39. Cameron, H.A. \& Gould, E. Adult neurogenesis is regulated by adrenal steroids in the dentate gyrus. Neuroscience 61, 203-209 (1994).

40. Heine, V.M., Maslam, S., Zareno, J., Joels, M. \& Lucassen, P.J. Suppressed proliferation and apoptotic changes in the rat dentate gyrus after acute and chronic stress are reversible. Eur. J. Neurosci. 19, 131-144 (2004).

41. Mirescu, C., Peters, J.D. \& Gould, E. Early life experience alters response of adult neurogenesis to stress. Nat. Neurosci. 7, 841-846 (2004).

42. Oomen, C.A. et al. Severe early life stress hampers spatial learning and neurogenesis, but improves hippocampal synaptic plasticity and emotional learning under high-stress conditions in adulthood. J. Neurosci. 30, 6635-6645 (2010).

43. Snyder, J.S., Soumier, A., Brewer, M., Pickel, J. \& Cameron, H.A. Adult hippocampal neurogenesis buffers stress responses and depressive behaviour. Nature 476, 458-461 (2011).

44. Seki, T. \& Arai, Y. Age-related production of new granule cells in the adult dentate gyrus. Neuroreport 6, 2479-2482 (1995).

45. Kuhn, H.G., Dickinson-Anson, H. \& Gage, F.H. Neurogenesis in the dentate gyrus of the adult rat: age-related decrease of neuronal progenitor proliferation. J. Neurosci. 16, 2027-2033 (1996).

46. van Praag, H., Christie, B.R., Sejnowski, T.J. \& Gage, F.H. Running enhances neurogenesis, learning, and long-term potentiation in mice. Proc. Natl. Acad. Sci. USA 96, 13427-13431 (1999).

47. Galea, L.A. Gonadal hormone modulation of neurogenesis in the dentate gyrus of adult male and female rodents. Brain Res. Rev. 57, 332-341 (2008).

48. Tanapat, P., Hastings, N.B., Reeves, A.J. \& Gould, E. Estrogen stimulates a transient increase in the number of new neurons in the dentate gyrus of the adult female rat. J. Neurosci. 19, 5792-5801 (1999).

49. Shors, T.J. et al. Neurogenesis in the adult is involved in the formation of trace memories. Nature 410, 372-376 (2001).

50. Leuner, B., Gould, E. \& Shors, T.J. Is there a link between adult neurogenesis and learning? Hippocampus 16, 216-224 (2006).

51. Deng, W., Aimone, J.B. \& Gage, F.H. New neurons and new memories: how does adult hippocampal neurogenesis affect learning and memory? Nat. Rev. Neurosci. 11, 339-350 (2010).

52. Bekinschtein, P., Oomen, C.A., Saksida, L.M. \& Bussey, T.J. Effects of environmental enrichment and voluntary exercise on neurogenesis, learning and memory, and pattern separation: BDNF as a critical variable? Semin. Cell Dev. Biol. 22, 536-542 (2011).

53. Dunnett, S.B., Wareham, A.T. \& Torres, E.M. Cholinergic blockade in prefrontal cortex and hippocampus disrupts short-term memory in rats. Neuroreport 1, 61-64 (1990).

54. Granon, S., Vidal, C., Thinus-Blanc, C., Changeux, J.P. \& Poucet, B. Working memory, response selection, and effortful processing in rats with medial prefrontal lesions. Behav. Neurosci. 108, 883-891 (1994).

55. Sloan, H.L., Good, M. \& Dunnett, S.B. Double dissociation between hippocampal and prefrontal lesions on an operant delayed matching task and a water maze reference memory task. Behav. Brain Res. 171, 116-126 (2006).

56. Chudasama, Y. \& Muir, J.L. A behavioural analysis of the delayed nonmatching to position task: the effects of scopolamine, lesions of the fornix and of the prelimbic region on mediating behaviours by rats. Psychopharmacology 134, 73-82 (1997).

57. Dudchenko, P. \& Sarter, M. Behavioral microanalysis of spatial delayed alternation performance: rehearsal through overt behavior, and effects of scopolamine and chlordiazepoxide. Psychopharmacology 107, 263-270 (1992).

58. Hearst, E. Delayed alternation in the pigeon. J. Exp. Anal. Behav. 5 225-228 (1962).

59. Herremans, A.H., Hijzen, T.H., Welborn, P.F., Olivier, B. \& Slangen, J.L. Effects of infusion of cholinergic drugs into the prefrontal cortex area on delayed matching to position performance in the rat. Brain Res. 711, 102-111 (1996).

60. McAllister, K.A.L., Saksida, L.M. \& Bussey, T.J. Dissociation between memory retention across a delay and pattern separation following medial prefrontal cortex lesions in the touchscreen TUNL task. Neurobiol. Learn. Mem. 101, 120-126 (2013).

61. Postman, L. \& Underwood, B. Critical issues in interference theory. Mem. Cogn. 1, 19-40 (1973).

62. Dale, R.H.I. \& Roberts, W.A. Variations in radial maze performance under different levels of food and water-deprivation. Animal Learn. Behav. 14, 60-64 (1986).

63. Roberts, W.A. \& Dale, R.H.I. Remembrance of places lasts-proactiveinhibition and patterns of choice in rat spatial memory. Learn. Motiv. 12, 261-281 (1981).

64. Grant, D.S. Intertrial interference in rat short-term memory. J. Exp. Psychol. Animal Behav. Processes 7, 217-227 (1981).

65. Nuechterlein, K.H. et al. The MATRICS consensus cognitive battery, part 1: test selection, reliability, and validity. Am. J. Psychiatry 165, 203-213 (2008).

66. Barch, D.M. et al. CNTRICS final task selection: working memory. Schizophr. Bull. 35, 136-152 (2009).

67. Harrison, P.J. \& Weinberger, D.R. Schizophrenia genes, gene expression, and neuropathology: on the matter of their convergence. Mol. Psychiatry 10, 40-68 (2005)

68. Harrison, P.J. The hippocampus in schizophrenia: a review of the neuropathological evidence and its pathophysiological implications. Psychopharmacology 174, 151-162 (2004).

69. Meyer-Lindenberg, A.S. et al. Regionally specific disturbance of dorsolateral prefrontal-hippocampal functional connectivity in schizophrenia. Arch. Gen. Psychiatry 62, 379-386 (2005).

70. Carter, C.S. et al. Functional hypofrontality and working memory dysfunction in schizophrenia. Am. J. Psychiatry 155, 1285-1287 (1998).

71. Creer, D.J., Romberg, C., Saksida, L.M., van Praag, H. \& Bussey, T.J. Running enhances spatial pattern separation in mice. Proc. Natl. Acad. Sci. USA 107, 2367-2372 (2010).

72. Coba, M.P. et al. TNiK is required for postsynaptic and nuclear signaling pathways and cognitive function. J. Neurosci. 32, 13987-13999 (2012).

73. Chaudhury, D. \& Colwell, C.S. Circadian modulation of learning and memory in fear-conditioned mice. Behav. Brain Res. 133, 95-108 (2002).

74. Beeler, J.A., Prendergast, B. \& Zhuang, X. Low amplitude entrainment of mice and the impact of circadian phase on behavior tests. Physiol. Behav 87, 870-880 (2006).

75. Roedel, A., Storch, C., Holsboer, F. \& Ohl, F. Effects of light or dark phase testing on behavioural and cognitive performance in DBA mice. Lab. Anim. 40, 371-381 (2006).

76. Satoh, Y., Kawai, H., Kudo, N., Kawashima, Y. \& Mitsumoto, A. Temperature rhythm reentrains faster than locomotor rhythm after a light phase shift. Physiol. Behav. 88, 404-410 (2006).

77. Bussey, T.J., Muir, J.L. \& Robbins, T.W. A novel automated touchscreen procedure for assessing learning in the rat using computer graphic stimuli. Neurosci. Res. Commun. 15, 103-110 (1994).

78. Cardinal, R.N. \& Aitken, M.R. Whisker: a client-server high-performance multimedia research control system. Behav. Res. Methods 42, 1059-1071 (2010). 